\title{
Physical forces in myelination and repair: a question of balance?
}

\author{
Nina G Bauer and Charles ffrench-Constant
}

\author{
Address: MRC Centre for Regenerative Medicine, Centre for Multiple Sclerosis Research, The University of Edinburgh, Queen's Medical \\ Research Institute, 47 Little France Crescent, Edinburgh EH16 4TJ, UK. \\ Correspondence: Nina G Bauer. Email: nina.g.bauer@ed.ac.uk
}

\begin{abstract}
A recent report in BMC Cell Biology examines how the balance of extracellular forces and intracellular contractions regulate the shape changes required for oligodendrocyte myelination. A failure of remyelination such as seen in multiple sclerosis could be caused by loss of this balance.
\end{abstract}

See related research article http://biomedcentral.com/1471-2121/10/71

\section{The interplay between intracellular and extracellular forces}

During development, all cells undergo enormous changes in cell shape. After a cell is 'born', it migrates to its final destination, where it then changes its shape to assume its final role. Very often, this involves the formation of cellular processes, many of which have specific shapes and functions that are characteristic to the individual cell types. This process outgrowth and other changes in morphology are supported internally by a sturdy network of specialized structural proteins that form the cytoskeleton. In addition, the surrounding extracellular environment, the extracellular matrix (ECM), mediates the changes in cell shape through its mechanical properties. The role of the ECM becomes particularly apparent when adherent cells (cells that are part of a tissue) are compared with non-adherent cells (cells that are floating freely within a liquid, such as blood). Although most adherent cells have a very particular shape, non-adherent cell types are usually rounded but change shape when they attach to surrounding tissue [1], suggesting that adherent cells can sense and respond to mechanical signals from the ECM.

These mechanosensory properties are mediated by special adhesion sites, where the ECM binds to a family of receptor proteins within the cell membrane. The most significant of these are the integrins. Binding of ECM ligands to integrins, with the associated varying degrees of mechanical strain or stretch, promotes the recruitment and linkage of part of the cytoskeleton, the actomyosin network, to intracellular integrin domains and thus anchors this network at the lipid membrane [2,3]. The actomyosin network consists of two major components, actin filaments and myosin molecules, which can slide along each other, creating an intracellular contractile force (Figure 1a). The relationship between this intracellular force and the strength of cell adhesion (the extracellular force) could then simply regulate shape such that stronger external forces would pull the cellular membrane outwards, whereas stronger internal forces would maintain a rounded shape.

However, cellular events seem to be more complex than this. First, recent findings have emphasized the importance of functional actomyosin contractile mechanisms for the regulation of a wide range of cell properties, including tissue formation, cell migration and cell differentiation [4]. Second, in contradiction to this simple model, low contractile forces generally yield membrane-rich, bulgy cell types, whereas strong contractions lead to the formation of highly structured cell shapes. Finally, different cell types reportedly have different ECM rigidity preferences for the induction of their particular shape. All this suggests that cellular shapes are determined by a precisely regulated interplay of intracellular contractile forces and extracellular attachment.

\section{Interplay of forces in myelination}

A particularly striking example of this interplay is the neural cell lineage, which gives rise to neurons, astrocytes and oligodendrocytes in the central nervous system (CNS). Developmentally, all three neural cell types develop from the same multipotent stem cells. However, neurons, which are generated first, prefer relatively soft surfaces for elaboration and branching of axons and dendrites. These softer substrates possibly correspond to the environmental conditions at the time of initial pathfinding of neuronal processes. In contrast, in recently published work in $B M C$ Cell Biology [5], Simons and colleagues show that the myelin-forming oligodendrocytes that develop later form their highly processed morphology and extensive myelin sheets best on more rigid surfaces.

This seems logical if we take a closer look at the developmental context of the formation of the insulating myelin sheath around axons. Once the migratory oligodendrocyte precursor cells (OPCs) have reached their destination and start to establish contact with an axon, their processes 
(a)

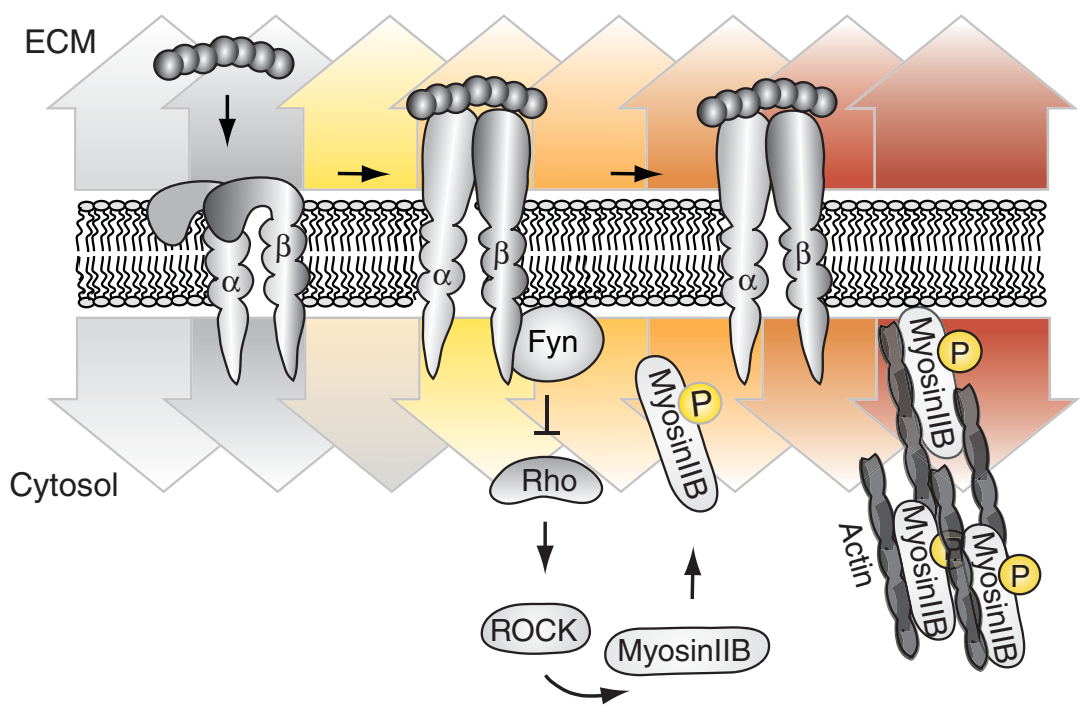

(b)

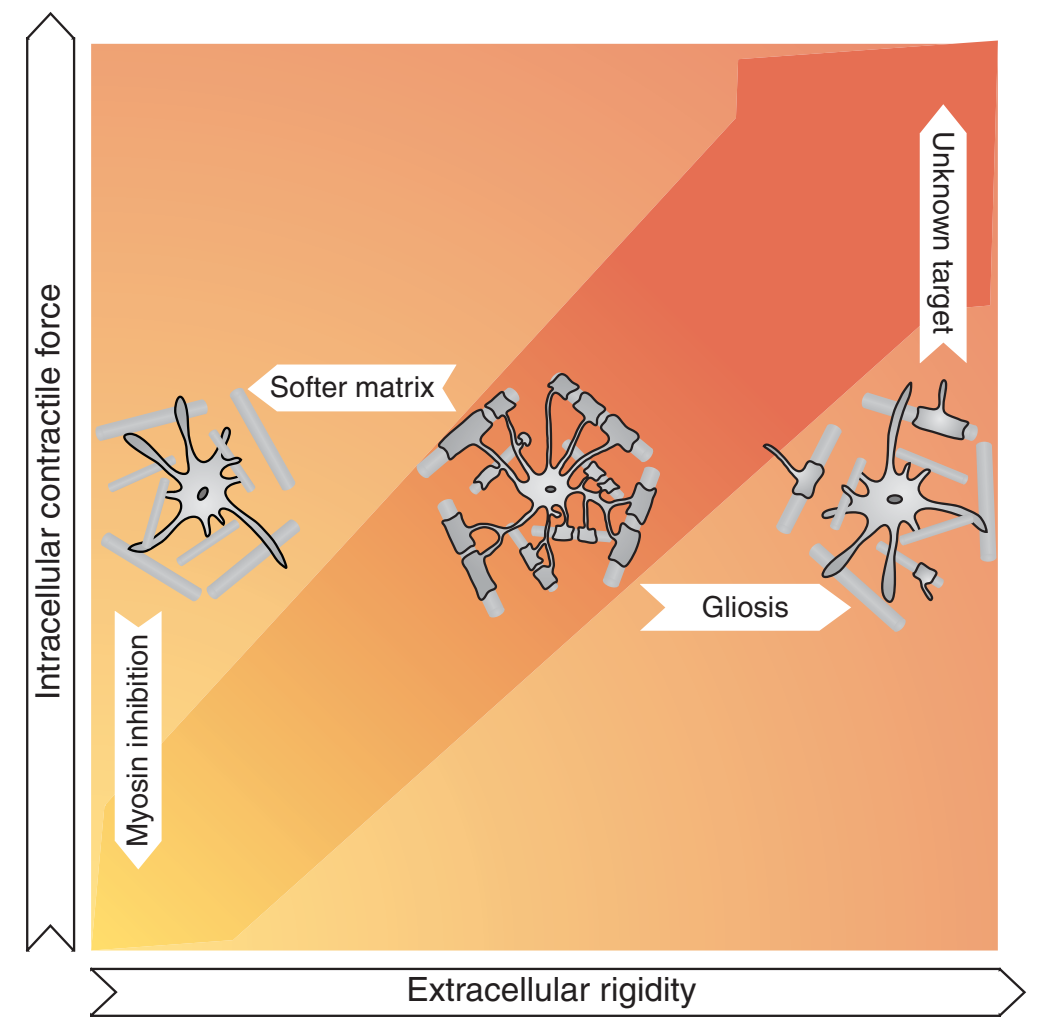

Figure 1

The role of force in myelination. (a) Molecular force generation. Cells bind ECM components through integrin receptors (represented by a and $\beta$ subunits), thus increasing extracellular adhesion. Integrin activation then triggers signaling cascades involving Fyn kinase, which inhibits RhoA, thus activating ROCK and Myosin IIB. Activated Myosin IIB interacts with actin filaments and creates strong intracellular contractions, which in turn enhances extracellular attachment and possibly mediates cell differentiation. ECM and cytosol color schemes represent the force intensity generated by these molecular events, gray being weakest and red being strongest. (b) Hypothetical effects of extracellular rigidity and intracellular contractions. Optimal myelination conditions require a balance between extracellular forces mediated by matrix rigidity and intracellular forces based on actomyosin contractions (diagonal arrow). A softer matrix inhibits cell differentiation and myelination (shift to the left), which can be counteracted by myosin IIB inhibition (cells return to being balanced). Gliosis, as it occurs in MS, might represent a more rigid matrix (shift to the right), which would require stronger contractile forces to counteract. 
change from exploring the unstructured extracellular environment of the presumptive myelinated tract, as required for migration, to the establishment of close contact with the highly structured (and therefore probably more rigid) surface of the axon, initiating the process of wrapping it with membranous sheets that will eventually become the compact myelin sheath [6]. An increase in intracellular force would therefore be necessary to enable the opposing forces to be matched and promote the next stage of oligodendrocyte development - the elaborate shape changes that accompany myelination (Figure $1 \mathrm{~b}$ ).

The findings of Simons and co-workers [5] also provide information about these intracellular mechanisms. Investigation of the role of intracellular contractility in differentiation and myelination identified myosin IIB, one of the major components of the actomyosin cytoskeleton, as a central player in generating intracellular force. In cell culture experiments, softer surfaces inhibit process outgrowth, as would be predicted if oligodendrocyte differentiation is normally associated with increased levels of intracellular force to match the increased rigidity of the axons. This effect can be overcome by pharmacological inhibition of myosin IIB, which will reduce intracellular contractions and thus better match intracellular force with the lower extracellular attachment efficacy provided by less rigid substrate (Figure 1b).

These findings are of particular interest for two reasons. The first is that they offer a clue as to how one might explain the rather surprising reported effects of myosin IIB inhibition on myelination in culture. Wang et al. [7] showed that myosin IIB inhibition in a neuron-oligodendrocyte co-culture system significantly enhanced the formation of the myelin sheath, a change that resulted from individual oligodendrocytes forming more wrapping processes than cells in untreated control cultures. In complete contrast, inhibition of myosin IIB in co-cultures of Schwann cells (the myelinating cells of the peripheral nervous system) with neurons inhibited myelination, and cellular morphology was characterized by aberrant process outgrowth. In short, while Schwann cells react as would be predicted, oligodendrocytes exhibit a behavior that contradicts the conclusions obtained from previous experiments. This might reflect important differences in the biology of the Schwann cell and the oligodendrocyte, in particular in respect to their adjustment to in vitro conditions: the extracellular forces on the Schwann cell appear to be similar in culture and in vivo, whereas the extracellular forces on oligodendrocytes in culture are potentially weaker than in vivo. The presence of a basal lamina on the non-axonal side of the Schwann cell but not the oligodendrocyte both in vitro and in vivo might be one means of retaining such an extracellular force.

\section{Interplay of forces in multiple sclerosis}

The second, and more important, reason for interest in the findings of Simons and colleagues [5] is that they offer explanations as to why remyelination might fail in the demyelinating disease multiple sclerosis (MS) [8]. In MS, unknown molecular triggers induce an inflammatory reaction in the brain leading to an invasion and activation of immune cells (B and T lymphocytes and macrophages) and/or the production of antibodies directed against myelin components. These events lead to the damage and degeneration of the myelin sheath. Remyelination does occur in the early stages of the disease as intrinsic mechanisms mediate the recruitment of OPCs, which then align with the denuded axon and regenerate the sheath. However, this repair mechanism eventually fails, for as-yet unknown reasons. An implication of the results of Simons and colleagues [5] is that increased rigidity in the scarred brain may play a role by unbalancing the intracellular and extracellular forces and inhibiting oligodendrocyte differentiation (Figure 1b).

How might the rigidity of the chronically demyelinated CNS be altered? Astrocytes, the third cell type derived from the neural lineage, provide nutrients to neurons and oligodendrocytes, give biochemical support to the cells forming the blood-brain barrier and, in particular, mediate the repair and scarring processes in the CNS following traumatic injuries. They respond to pathological insults, including inflammation and demyelination, with so-called reactive gliosis. On a cellular level, this is characterized by an upregulation of intermediate filament proteins, leading to the formation of a prominent intermediate filament network directly underneath the plasma membrane, rendering the cellular texture more fibrous [9]. Furthermore, pronounced changes in expression of adhesion molecule genes have been described, which would result in an altered ECM composition compared with that of initial myelination. In demyelinated plaques, reactive astrocytes are the most abundant cellular component, and astroglial scars have been described as being more rigid than their surrounding tissue. These properties might alter forcesensing integrin function in the oligodendrocyte, unbalancing the cellular forces and inhibiting remyelination.

The main implications of the findings of Simons and colleagues [5] are, therefore, that a particular balance of extracellular adhesion, matrix rigidity and intracellular contractile forces mediated by the oligodendrocyte actomyosin cytoskeleton is required for successful myelination and remyelination. One interesting prediction implied by these data is that extracellular cues that do not in themselves alter rigidity, but that do change the activity of signaling molecules regulating intracellular force, could also inhibit remyelination. As discussed above, the predominant pathway involved in the signaling mechanisms underlying mechanosensing and mechanotransduction is 
binding of ECM ligands to integrin receptors in the membrane. The activation of integrins by mechanical forces results in the recruitment of intracellular mediators that signal through a pathway involving RhoA and its downstream effector ROCK to activate force-generating myosin II (Figure 1a). The observation that the inhibitory effects of myelin debris on OPC differentiation, myelination and remyelination are mediated by RhoA-ROCK signaling [10] is consistent with this hypothesis [5]. Subsequent pharmacological disruption of the ROCK pathway, inhibiting myosin IIB and thus actomyosin contractility, was able to enhance oligodendrocyte differentiation [10]. Clearly, the signaling molecules that regulate intracellular force now provide an intriguing source of candidates for drug discovery programs aimed at enhancing remyelination (Figure 1b).

\section{References}

1. Discher DE, Mooney DJ, Zandstra PW: Growth factors, matrices, and forces combine and control stem cells. Science 2009, 324:1673-1677.

2. Choquet D, Felsenfeld DP, Sheetz MP: Extracellular matrix rigidity causes strengthening of integrin-cytoskeleton linkages. Cell 1997, 88:39-48.

3. Schewkunow V, Sharma KP, Diez G, Klemm AH, Sharma PC, Goldmann WH: Thermodynamic evidence of non-muscle myosin II-lipid-membrane interaction. Biochem Biophys Res Commun 2008, 366:500-505.

4. Clark K, Langeslag M, Figdor CG, van Leeuwen FN: Myosin II and mechanotransduction: a balancing act. Trends Cell Biol 2007, 17:178-186.

5. Kippert A, Fitzner D, Helenius J, Simons M: Actomyosin contractility controls cell surface area of oligodendrocytes. BMC Cell Biol 2009, 10:71.

6. Bauer NG, Richter-Landsberg C, ffrench-Constant C: Role of the oligodendroglial cytoskeleton in differentiation and myelination. Glia 2009, doi: 10.1002/glia.20885.

7. Wang H, Tewari A, Einheber S, Salzer JL, MelendezVasquez CV: Myosin II has distinct functions in PNS and CNS myelin sheath formation. J Cell Biol 2008, 182:11711184.

8. Franklin RJ, ffrench-Constant $\mathrm{C}$ : Remyelination in the CNS: from biology to therapy. Nat Rev Neurosci 2008, 9:839-855.

9. Williams A, Piaton G, Lubetzki C: Astrocytes - friends or foes in multiple sclerosis? Glia 2007, 55:1300-1312.

10. Baer AS, Syed YA, Kang SU, Mitteregger D, Vig R, ffrenchConstant C, Franklin RJ, Altmann F, Lubec G, Kotter MR: Myelin-mediated inhibition of oligodendrocyte precursor differentiation can be overcome by pharmacological modulation of Fyn-RhoA and protein kinase C signalling. Brain 2009, 132:465-481.

Published: 25 September 2009

doi:10.1186/jbiol169

(c) 2009 BioMed Central Ltd 